\title{
Integrin-linked kinase: A hypoxia-induced anti-apoptotic factor exploited by cancer cells
}

\author{
ELIZABETH R. ABBOUD ${ }^{1}$, SETH B. COFFELT ${ }^{1}$, YANIRA G. FIGUEROA ${ }^{2}$, KEVIN J. ZWEZDARYK ${ }^{2}$, \\ ANNE B. NELSON ${ }^{2}$, DEBORAH E. SULLIVAN ${ }^{3}$, CINDY B. MORRIS ${ }^{2}$, YAN TANG $^{4}$, \\ BARBARA S. BECKMAN ${ }^{4}$ and ALINE B. SCANDURRO ${ }^{2}$ \\ ${ }^{1}$ Molecular and Cellular Biology Program, Departments of ${ }^{2}$ Microbiology and Immunology, ${ }^{3}$ Pathology, \\ and ${ }^{4}$ Pharmacology, Tulane University School of Medicine, New Orleans, LA, USA
}

Received August 3, 2006; Accepted September 19, 2006

\begin{abstract}
Based on cDNA microarray results, integrin-linked kinase (ILK) emerged as an interesting candidate in hypoxiamediated survival mechanisms employed by cancer cells. This notion was confirmed here by the following observations: the 5' promoter region of the ilk gene contains hypoxia responsive elements (HRE) that bind hypoxia-inducible factor (HIF) transcription factor complexes and drive HRE-luciferase gene expression in reporter assays; ILK protein and kinase activity are induced following hypoxia; downstream targets of ILK signaling are induced following hypoxia treatment; inhibition of ILK leads to increased apoptosis; and HIF and ILK are co-localized within human cancer tissues. The identification of ILK as a player in hypoxia survival signaling employed by cancer cells further validates ILK as a unique target for cancer therapy.
\end{abstract}

\section{Introduction}

Established hypoxia or low oxygen within a tumor is a known instigator of the malignant progression of many human cancers. To determine some of the critical signaling pathways in hypoxia induced anti-apoptotic mechanisms employed by tumor cells, human hepatocellular carcinoma or Hep3B cells

Correspondence to: Dr Aline B. Scandurro, Department of Microbiology and Immunology, Tulane University Health Sciences Center, 1430 Tulane Ave., SL38, New Orleans, LA 70112, USA

E-mail: alibscan@tulane.edu

Abbreviations: ILK, integrin-linked kinase; HRE, hypoxia responsive element; HIF, hypoxia-inducible factor; VEGF, vascular endothelial cell growth factor; PI3K, phosphotidylinositol-3-kinase; PKB, protein kinase B; GSK, glycogen synthase kinase; PTEN, phosphatase and tensin homolog deleted on chromosome 10; ECM, extracellular matrix; Epo, erythropoietin; DFO, desferrioxamine; $\mathrm{CoCl}$, cobaltous chloride; $\mathrm{RCC}$, renal carcinoma cell

Key words: integrin-linked kinase, hypoxia, low oxygen, apoptosis, cell signaling were subjected to low oxygen $\left(1 \% \mathrm{O}_{2}\right)$ and analyzed by microarray cDNA analyses. From this approach, integrinlinked kinase (ILK) emerged as an interesting candidate for hypoxia-induced anti-apoptotic mechanisms employed by cancer cells (1). Integrin-linked kinase is a serine and threonine protein kinase containing four ankyrin-like repeats and a putative phosphoinositide phospholipid-binding motif. ILK was so named because it interacts with the $\beta$-chain cytoplasmic domains of integrins serving to localize this kinase to focal adhesion plaques (2). Both cell-fibronectin interaction and insulin treatments affect ILK activity. It can be regulated by the established pro-survival kinase, phosphotidylinositol 3-kinase (PI3K) and subsequently leads to phosphorylation of protein kinase B (PKB/Akt-ser473) and glycogen synthase kinase (GSK-3 ser9), both signaling molecules upstream of several transcription factors including LEF-1 and AP-1 $(3,4)$. As a result of some of these activities, ILK has been critically implicated in a diverse number of complex cellular processes including anchorage-independent cell survival, cell cycle progression, inflammation, epithelial-mesenchymal transitions and tumorigenesis in nude mice (2).

The ability of ILK to directly phosphorylate substrates, $\mathrm{PKB} /$ Akt and/or GSK and whether this is important for its many physiological roles has been disputed in the past (5-7). Work in lower eukaryotes has demonstrated that ILK functions not as a kinase but as a docking or adaptor protein downstream of integrin receptors. Conversely, work in mammalian cell systems has concentrated primarily on ILK kinase overexpression and its effect on tumorigenesis. One significant feature of ILK is that it represents a unique convergence point in cell signaling from both growth factors, such as insulin, and extracellular matrix components, such as fibronectin. It has been reported that overexpression of ILK in epithelial cells leads to an increase in fibronectin matrix assembly that in turn reduces cellular E-cadherin expression and ultimately leads to increased tumor formation in nude mice (8). Several investigators have now shown a correlation between ILK overexpression and cancer. In one report, increased ILK expression correlated with increased prostate tumor grade (9). In another, ILK overexpression was suggested as a potential marker in diagnosis of Ewing's sarcoma and other primitive neuroectodermal cancers (10). ILK-2 a subsequently cloned 
isoform of ILK (different by only four amino acids) is expressed only in metastatic human melanomas and other metastatic cancer cell lines but not in non-invasive melanomas or normal adult tissues (11). Finally, ILK activation upstream of key anti-apoptotic PKB/Akt cell signaling pathways has been identified as a critical target in PTEN tumor suppressordependent tumor therapies. This suggestion comes from studies showing that ILK expression is constitutively elevated in PTEN-mutant cancer cell lines. Additionally, transfection of prostate carcinoma cell lines with a dominant negative form of ILK leads to cell cycle arrest and apoptosis of the cancer cells. Equal results were observed if the wild-type tumor suppressor PTEN was reintroduced by transfection into PTENnull cancer cells. From these results, a critical role for ILK in PTEN-mediated cell cycle regulation and survival was suggested (12). In recent reports, ILK was clearly mapped out as a key survival factor for keratinocytes that prevents apoptosis induced by gangliosides by serving to activate $\mathrm{PKB} / \mathrm{Akt}$ that then inhibits pro-apoptotic events including mitochondrial release of cytochrome $\mathrm{c}$ and sub-sequent caspase 9 activation (13). Intriguingly, inhibition of ganglioside-induced apoptosis by ILK-mediated pathways was determined to follow the ECM-integrin signaling pathway rather than growth factor signaling pathway of ILK. ILK clearly represents an unexplored yet critical target in many human cancers as has recently been suggested and is gleaned from the results presented here (14). However, the molecular details of how the commonly noted tumor-associated hypoxia might affect ILK expression and activity have not been addressed.

In this study, the hypothesis that integrin-linked kinase is hypoxia-regulated and serves as a critical regulator of antiapoptotic mechanisms exploited by cancer cells was tested. The novel identification of ILK as a player in low oxygen or hypoxia anti-apoptotic signaling employed by cancer cells further validates ILK as a unique target for cancer therapy (14).

\section{Materials and methods}

Cell culture. Human hepatocellular carcinoma cells, Hep3B, were purchased from the American Type Tissue Collection (VA) and maintained in Eagle's minimal essential medium (Invitrogen Corp., CA) supplemented with penicillin (100 U/ $\mathrm{ml})$, streptomycin $(100 \mu \mathrm{g} / \mathrm{ml}), 0.1 \mathrm{mM}$ non-essential amino acids, $0.2 \mathrm{mM}$ glutamine, $1 \mathrm{mM}$ pyruvate, and $10 \%$ fetal bovine serum (FBS) and incubated in a $95 \%$ air $/ 5 \% \mathrm{CO}_{2}$ humidified atmosphere at $37^{\circ} \mathrm{C}$. Human renal carcinoma cells, RCC-786-O, were a generous gift from Dr James Gnarra (Louisiana State University, LA) and maintained in Dulbecco's modified Eagle's medium (DMEM) with supplements of penicillin $(100 \mathrm{U} / \mathrm{ml})$, streptomycin $(100 \mu \mathrm{g} / \mathrm{ml}), 0.1 \mathrm{mM}$ non-essential amino acids, $0.2 \mathrm{mM}$ glutamine, $1 \mathrm{mM}$ pyruvate, and $10 \%$ fetal bovine serum (FBS) and incubated in a $95 \%$ air $/ 5 \% \mathrm{CO}_{2}$ humidified atmosphere at $37^{\circ} \mathrm{C}$. Hypoxia exposure was carried out by placing the cell cultures in an oxygen controlled chamber maintained humidified at $37^{\circ} \mathrm{C}$ with settings at $1 \%$ oxygen $\left(5 \% \mathrm{CO}_{2}\right.$ and balance $\mathrm{N}_{2}$ ) (Coy Laboratories, $\left.\mathrm{MI}\right)$.

Plasmids and vectors. pILK-wt and pILK-KD (E359K) were kindly provided by the laboratory of Dr Shoukat Dedhar (University of British Columbia, Vancouver, Canada) and were prepared from human sequences of ILK cloned into pcDNA3.1 (Invitrogen) as described previously (15). The full-length promoter construct for ILK, (pILK-Pr) and 3-phosphoinositidedependent kinase-1 (PDK, pPDK-Pr) containing two HRE and no HRE, respectively, as well as a peroxisome proliferatoractivated receptor response element (PPRE) driving the luciferase gene were generous gifts from the laboratory of Dr Beatrice Desvergne (Universite de Lausanne, Switzerland) and have been previously described (16). Adenovirus vectors containing either ILK-wt (Ad-ILK-wt) or ILK-KD (Ad-ILKKD) genes were developed in the present study (AdEasy vector system, Stratagene, CA). For this purpose, the pILK-wt and pILK-KD genes were subcloned into pShuttle-CMV and by double homologous combination further cloned into an adenovirus (Ad5) backbone pAdEasy-1 (as per manufacturer's instructions).

EMSA. Cells were washed with ice-cold PBS and harvested by scraping in $1.5 \mathrm{ml}$ of PBS. Cells were pelleted and resuspended in $400 \mu \mathrm{l}$ of buffer A [10 mM HEPES (pH 7.9), $1.5 \mathrm{mM} \mathrm{MgCl}_{2}$, $10 \mathrm{mM} \mathrm{KCl}, 0.5 \mathrm{mM}$ DTT, $0.2 \mathrm{mM}$ PMSF]. After $10 \mathrm{~min}$ of incubation on ice, nuclei were pelleted and then resuspended in $50 \mu 1$ of buffer B [20 mM HEPES-KOH ( $\mathrm{pH} 7.9$ ), 25\% glycerol, $420 \mathrm{mM}$ sodium chloride, $1.5 \mathrm{mM} \mathrm{MgCl}_{2}, 0.2 \mathrm{mM}$ EDTA, $0.5 \mathrm{mM}$ DTT, $0.2 \mathrm{mM}$ PMSF]. Proteins were incubated for $20 \mathrm{~min}$ on ice and then centrifuged to clear cellular debris. Gel shift assays were performed by incubating $2 \mu \mathrm{g}$ of the nuclear extracts in gel shift binding buffer (cat\#E3300, Promega Corp., WI) for $20 \mathrm{~min}$ at room temperature with the $\left[\gamma^{-32} \mathrm{P}\right]$-labeled oligonucleotides (50 fmol, 50-200,000 counts/ min were loaded per lane). The protein-DNA complexes were analyzed by electrophoresis through a $5 \%$ polyacrylamide gel, with $0.5 \mathrm{X}$ Tris/borate/EDTA buffer (TBE: $0.045 \mathrm{M}$ Tris borate, $0.001 \mathrm{M}$ EDTA) and $3.5 \%$ glycerol at $180 \mathrm{~V}$ for $30 \mathrm{~min}-1 \mathrm{~h}$. The gels were dried and exposed to autoradiography film or phosphoimaging plates.

Luciferase assays. Hep3B cells were seeded to $70 \%$ confluence in $10-\mathrm{cm}$ culture dishes before liposomal mediated transient transfection with Fugene6 (Roche Molecular Biochemicals, IN) with test firefly luciferase plasmid vectors (500 ng) and Renilla luciferase control vector (5 ng, Dual-light reporter gene assay system, Promega). Following overnight recovery, the cultures were transferred to the hypoxia $\left(1 \% \mathrm{O}_{2}\right)$ chamber or treated with $100 \mu \mathrm{M} \mathrm{CoCl}$ (Sigma Aldrich, MO) or $100 \mu \mathrm{M}$ desferrioxamine (Sigma Aldrich) for the indicated times. Immediate cell lysis was achieved at time of harvest by addition of lysis buffer (Dual-light reporter gene assay system, Promega). Luciferase activity of treated cell extracts was measured following the manufacturer's instruction in a Turner Designs TD20/20 Luminometer (Dual-light reporter gene assay system, Promega).

Western blot analysis (17). After separation on SDS-PAGE, proteins were transferred to nitrocellulose. Nitrocellulose membranes were then blocked with 5\% Blotto in TBSTween-N20 buffer and incubated with antibodies as indicated in figure legends. Subsequent to HRP-linked IgG blotting, the blots were visualized by chemiluminescence (ECL, Amersham Biosciences, NJ). 
ILK kinase assay (18). Cells were lysed in hypotonic buffer as per manufacturer's recommendation (mammalian protein extraction reagent, M-PER, Pierce Biotechnology, IL). ILK was immunoprecipitated from the cell lysates and incubated in vitro with myelin basic protein (MBP) as the ILK kinase substrate in the presence of radiolabeled $\left[\gamma_{-}{ }^{32} \mathrm{P}\right]-\mathrm{ATP}$ used as the donor. The reaction was analyzed following separation of protein on $12 \%$ SDS-PAGE and autoradiography or phospho-imaging. A duplicate gel was also transferred to nitrocellulose for Western blot analysis to measure input kinase in each immunoprecipitated sample as outlined above.

Apoptosis assays. ApoAlert Annexin V kit was used to detect early apoptotic events by flow cytometry as per manufacturer's recommendation (cat\#K2013-1, BD, Clontech, CA). Uninduced, and unstained, induced controls were always analyzed in parallel to test samples as recommended. Transfected or transduced Hep3B cells were exposed to hypoxia for $0,6,24$ and $48 \mathrm{~h}$ before harvesting both spent culture medium and scraped cells in a conical tube and pelleting at low speed prior to labeling with Annexin V-PE for $15 \mathrm{~min}$ with gentle agitation at room temperature. Flow cytometry was performed on a BD-FACScalibur and analyzed with CellQuest Pro software (ver. 3.5). The APO-BrdU TUNEL assay kit was used to measure later apoptotic events (cat\#A-23210, Molecular Probes, OR). Cells were grown on chamber slides to $50-70 \%$ confluency prior to treatments or hypoxia exposure for $0,12,24,48,72$ and $96 \mathrm{~h}$. Cells were fixed and permeabilized with cytofix/cytoperm kit (BD, Pharmigen, MA) prior to BrdU DNA-labeling and Alexa Fluor 488 dye-labeled anti-BrdU antibody detection as per manufacturer's instructions. All nuclei were visualized following propidium iodide/RNase A staining as recommended on a Zeiss Axioplan 2 Fluorescent Microscope with SlideBook ${ }^{\mathrm{TM}} 4.0$ (Intelligent Imaging Innovations Deconvolution Hardware and Software, Denver, CO).

Immunohistochemical analysis. Formalin-fixed paraffinembedded human tissue arrays available for immunohistochemical (IHC) analysis were obtained (cat\#T8235714D, Biochain Institute Hayward, CA) and probed with the ILK polyclonal antibody (1:50, cat\#06-592, Upstate Biotechnologies, NY) and HIF-1 $\alpha$ polyclonal antibody (1:200, cat\#sc12542, Santa Cruz Biotechnologies, CA) for determination of the expression of each of these proteins in a variety of human normal (top panels) and cancerous (bottom panels) tissues, including placenta, lung, bladder, breast, kidney, ovary and uterus. The tissue biopsy array used was composed of 10 tumor biopsies and 1 matched normal sample derived from human bladder, breast, kidney, lung, ovary and uterus tissues. Immunohistochemical staining was achieved with the horseradish peroxidase enhancement method of the LSAB 2 kit (DakoCytomation Corp, CA). Micrographs were taken on a Zeiss Axioplan 2 Fluorescent Microscope with Intelligent Imaging Innovations Deconvolution Hardware and Software (SlideBook ver. 4).

Statistical analysis. Data are shown as standard error of the mean (SEM). Multiple group comparison was performed by one-way analysis of variance (ANOVA) followed by the

\section{Human ILK gene}

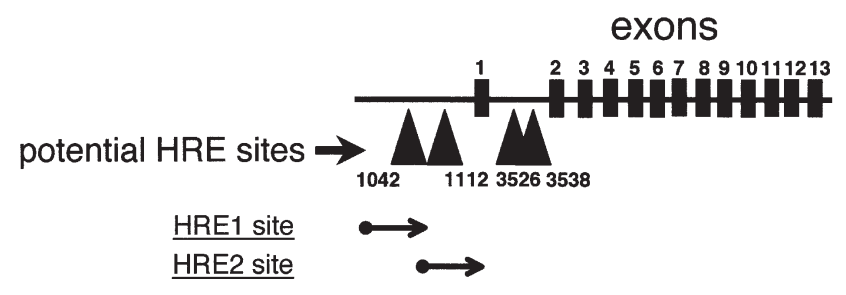

Figure 1. Schematic representation of the human integrin-linked kinase (ILK) gene. Solid triangles represent the locations of some of the potential hypoxia responsive elements (HRE) found within the published sequence (GenBank accession no. AJ404847). Closed ended arrows represent the sites analyzed in this study, ILK-HRE1 and -2.

Bonferroni procedure for comparison of means. Comparison between two groups was analyzed by the two-tailed Student's t-test or two-way ANOVA (Prism4, GraphPad Software Inc. CA). Values of $\mathrm{P}<0.05$ were considered statistically significant.

\section{Results}

Integrin-linked kinase gene expression is regulated by hypoxiainducible factor (HIF). Microarray (cDNA) analysis was used to gain a better understanding of the molecular factors critical for low oxygen survival in the Hep3B cell model system (1). Changes in the RNA expression of several genes were detected including those of known oxygen-responsive genes, such as erythropoietin (Epo) and hypoxia-inducible factor-1 (HIF-1) that served to validate successful low oxygen $\left(1 \% \mathrm{O}_{2}\right)$ treatment of the cells. From this approach, integrinlinked kinase (ILK) emerged as a novel hypoxia-responsive gene candidate. Increased expression of ilk after hypoxia might be explained by transactivation of the ilk gene by HIF. HIF are a family of nuclear heterodimeric ( $\alpha$ and $\beta$ ) transcription factors responsible for induction of several genes following hypoxia exposure of mammalian cells (19). Based on computer analysis of the published ilk gene (GenBank no. AJ404847) at least four potential consensus HIF binding sites (or hypoxia responsive elements, HRE, RCGTG) for ILK were identified (Fig. 1, MacVector ver. 6.5.3, Oxford Molecular). The HRE found within the ilk gene lie 5' to exon 1 as well as between exon 1 and 2 (nt 1042-7, 1107-1112, 3526-31, 3533-8). Electrophoretic mobility shift assays (EMSA) were performed with nuclear extracts derived from hypoxia-treated cells to test the ability of the identified potential HRE of ilk to bind HIF protein complexes (labeled here as ILK-HRE1 and -HRE2). Oligonucleotides spanning two ilk HRE as well as the control erythropoietin (Epo) enhancer HRE (EpoE HRE) were prepared commercially (Integrated DNA Technologies, IA) and radiolabeled with polynucleotide kinase (Invitrogen). HRE were originally identified and characterized from the Epo enhancer region that was used here as a positive control. As seen in a representative autoradiogram both ilk HRE appear to bind protein complexes that migrated similar to control HIF binding EpoE HRE (Fig. 2A). No shifted protein complex was observed in the absence of protein. Additionally, formation of EpoE HRE- 

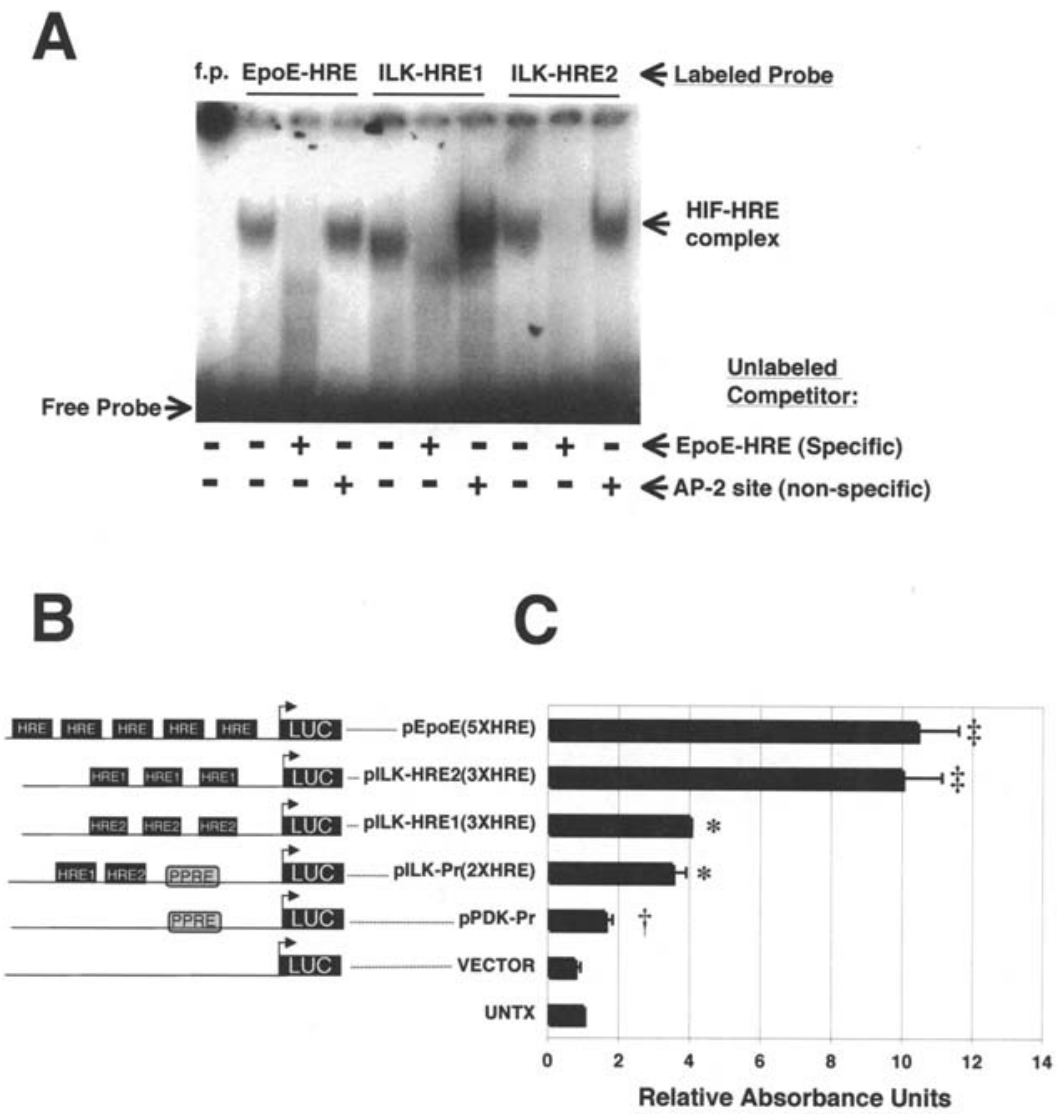

Figure 2. Similar to the canonical erythropoietin enhancer HRE, the ILK HRE form HIF protein complexes and are transactivated by hypoxia exposure. Analysis of the hypoxia responsive elements (HRE) found within the human integrin-linked kinase (ILK) gene demonstrates properties similar to the known hypoxia responsive gene sequences of the erythropoietin gene. A, HIF heterodimer-ILK-HRE complexes form. EMSA was performed using Hep3B cell nuclear extracts and oligonucleotide probes as indicated. The first lane represents free probe (f.p.) without protein extract. Labeled probes used in the reactions with the treated nuclear extracts are indicated above the lanes. Specific competition was achieved with 100-fold molar excess of unlabeled oligonucleotides of EpoE-HRE. Non-specific competition was achieved with 100-fold molar excess of the unrelated AP-2 site encoding oligonucleotides as indicated. Arrow denotes specific complexes between HIF heterodimer and the oligonucleotide probes. B, schematic representation of the luciferase reporter vectors analyzed including the number of HRE found within each construct. The erythropoietin gene luciferase construct, pEpoE has five. The test integrin-linked kinase constructs, pILK-HRE1, -HRE2 each have three. The full-length promoter constructs for ILK, pILK-Pr and 3-phosphoinositide-dependent kinase-1 (PDK), pPDK-Pr have two and no HRE, respectively as shown, as well as a peroxisome proliferator-activated receptor response element, PPRE. C, transfected cells $(500 \mathrm{ng})$ were allowed to recover prior to overnight exposure to hypoxia $\left(1 \% \mathrm{O}_{2}-18 \mathrm{~h}\right)$. Results were normalized following simultaneous transfection and analysis of a Renilla luciferase reporter vector (5 ng, Dual-Luciferase Assay kit, Promega) and are expressed as relative absorbance units to the measured activity of the empty promoter luciferase vector (Vector). Values represent means of triplicate samples from at least four independent experiments. Statistical analysis was performed with Prism 4.0 (GraphPad Software Inc.). ${ }^{*} \mathrm{P}<0.001 ;{ }^{*} \mathrm{P}<0.01$; and ${ }^{\dagger} \mathrm{P}>0.05$ over vector or untreated (UNTX) control samples.

and both ILK HRE-shifted protein complexes is impeded by the addition of 100 -fold molar excess of unlabeled EpoE HRE (shown) or by addition of either of the unlabeled ILK HRE (data not shown) indicating that EpoE HRE and ILK HRE compete for the same HIF protein complex. In contrast, competition with a non-specific unlabelled AP-2 probe did not affect EpoE HRE or either of the ILK HRE protein complexes as shown. These results strongly implicate HIF proteins specifically binding to the ILK HRE.

To further demonstrate that ilk is regulated by HIF transcription factors, the ilk HRE were subcloned into a promoter-containing luciferase reporter vector (pGL3-promoter vector, Promega). Three copies of each ilk HRE1 and ilk HRE2 were subcloned into this vector as confirmed by nucleotide sequencing of the resulting plasmids (Fig. 2B). Hep3B cells were transiently transfected with either the empty vector (pGL3-promoter), a positive control vector containing five copies of the EpoE-HRE, the newly cloned ILK-HRE1 vector or the newly cloned ILK-HRE2 vector.
Following an 18-h recovery period the transfected cells were treated with normal air $\left(21 \% \mathrm{O}_{2}\right)$, hypoxia $\left(1 \% \mathrm{O}_{2}\right)$ or other known hypoxia mimetic agents such as cobaltous chloride $(\mathrm{CoCl}, 100 \mu \mathrm{M}$ ) or desferrioxamine (DFO, $100 \mu \mathrm{M}$ ) for $18 \mathrm{~h}$. The resulting luciferase activity was tested after sample lysis as recommended by the manufacturer (Dual-Luciferase Assay kit, Promega). Normalization of the samples was achieved following co-transfection of all the samples with a control Renilla luciferase vector (5 ng, pRL-SV40) as per manufacturer's instruction (Dual-Luciferase Assay kit, Promega). The results indicate that like the positive control EpoE HRE, the ILK HRE can be transactivated following hypoxia exposure or following treatment with $\mathrm{CoCl}$ or DFO (Fig. 2C). The empty vector or untreated control had no appreciable transactivation following any of the treatments. The full-length promoter construct for ILK (pILK-Pr) and 3-phosphoinositidedependent kinase-1 (PDK, pPDK-Pr) containing two and no HRE, respectively, as well as a peroxisome proliferatoractivated receptor response element (PPRE) were also tested 
A
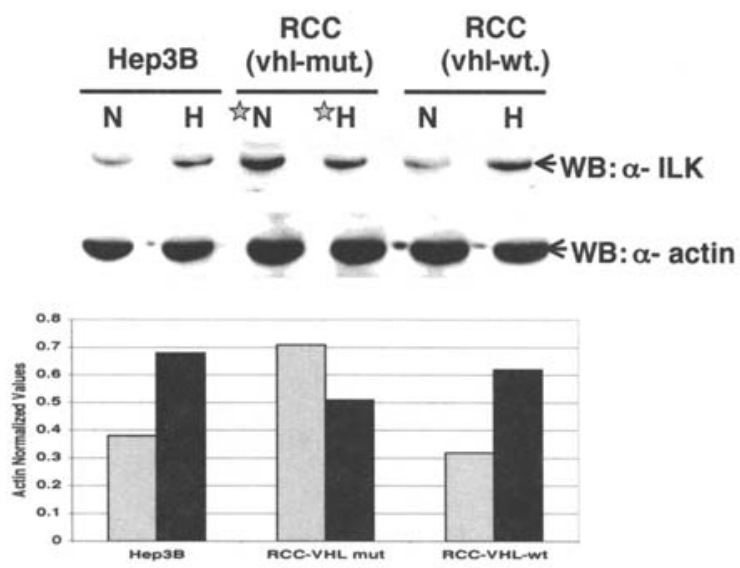

B

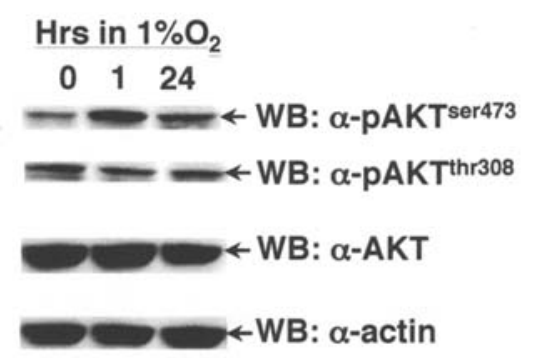

C

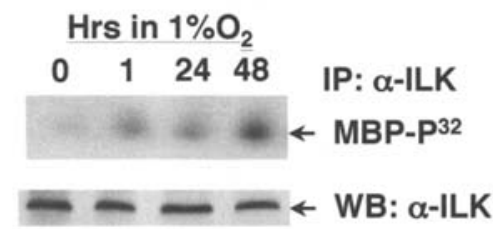

Figure 3. ILK protein expression and kinase activity is affected by hypoxia exposure of cells. A, total cellular lysates $(30 \mu \mathrm{g})$ from Hep3B or human renal carcinoma cells (RCC) expressing the wild-type or mutant von Hippel-Lindau (vhl) protein (vhl-wt. or vhl-mut, respectively) exposed to hypoxia for $6 \mathrm{~h}$ were analyzed by Western blotting (top panel). Blots were probed with the antibodies indicated by arrows, (WB:) ILK (1:5,000, Upstate Biotechnology Inc.) and actin (1:10,000, Sigma-Aldrich, MO). N, denotes normoxia-treated cells; $\mathrm{H}$, cells treated for $6 \mathrm{~h}$ in $1 \% \mathrm{O}_{2}$. Bottom graph represents the plotted densitometric values that resulted after normalization with $B$-actin blot. B, Western blot analysis of Hep3B cells exposed to $1 \% \mathrm{O}_{2}$ for the times indicated with phospho-specific antibodies for protein kinase B/AKT residues serine 473 (pAKT $\left.^{\mathrm{ser} 473}\right)$ or threonine 308 (pAKT ${ }^{\text {thr308 }}, 1: 1,000$, Cell Signalling) for ILK downstream signaling molecules and compared with total AKT (1:1,000, Cell Signalling) or $B$-actin. C, ILK kinase is stimulated by exposure to hypoxia. Cell lysates from Hep3B cells exposed to $1 \% \mathrm{O}_{2}$ for the times indicated were analyzed for ILK kinase activity following immunoprecipitation (IP) with ILK antibody and using myelin basic protein (MBP) as the ILK substrate as described (18). Total ILK contained in the kinase assay reaction mixtures are demonstrated by companion Western blot (WB) analysis of the same lysates with the ILK antibody as described above. Results are representative of at least four experiments performed with independently derived lysates.

(Fig. 2C). Transfection of the Hep3B cells with pILK-Pr consistently resulted in modest hypoxia-, CoCl- or DFOmediated induction of the promoter region when compared with empty vector controls or the related PDK1 promoter containing luciferase vectors. The sum of these observations strongly supports the concept that HIF specifically regulates ilk HRE during low oxygen or hypoxia exposure.
Expression of integrin-linked kinase in hypoxia-exposed cancer cells. The effect of hypoxia on integrin-linked kinase protein expression in cancer cells was tested by Western blot analysis (Fig. 3A). Hep3B cells and renal carcinoma cells (RCC) were exposed to hypoxia $\left(1 \% \mathrm{O}_{2}\right)$ for $6 \mathrm{~h}$. Hep3B cells are the test hypoxia tolerant model system, renal carcinoma cells (786-O, pVHL mutant generously provided by the laboratory of Dr James Gnarra) were used because they contain a known defect in a tumor suppressor gene named von Hippel-Lindau ( $v h l$ ) that leads to dysregulated expression of all HIF controlled genes. The VHL protein ( $\mathrm{VVHL}$ ) is responsible for specifically polyubiquitinating and targeting the $\alpha$ subunit of HIF for degradation in normal oxygenated conditions. During hypoxia, the pVHL recognition motif on HIF is modified; pVHL no longer binds and HIF is allowed to hetereodimerize, travel to the nucleus and transactivate its target genes $(20,21)$. In the RCC-786-O cells, because of the $v h l$ defect all HIF-regulated genes are induced regardless of exposure to normal room air $\left(21 \% \mathrm{O}_{2}\right)$ or hypoxia $\left(1 \% \mathrm{O}_{2}\right)$ (22). By contrast in the RCC-wt cells, where the normal vhl has been re-introduced, HIF-regulated genes are only induced following hypoxia as in wild-type cells. Thus, if $i l k$ is regulated by HIF, ILK protein expression should be induced by hypoxia in Hep3B cells and RCC-wt where normal HIF control is present. However, ILK protein expression should be induced regardless of treatment in the RCC-786-O (pVHL-mut) where there is no HIF control. The representative immunoblot autoradiogram shows that that is exactly what was observed, providing further evidence that ILK is hypoxia-regulated (Fig. 3A). The actin antibody probed immunoblot is also included below as reference since densitometric analysis of this blot was used to normalize the ILK-probed blot represented in the bar graph shown.

A distinct role for ILK in low oxygen responses was also implicated from evidence of specific activation of protein kinase B (AKT) a downstream target of ILK, following hypoxia treatment of Hep3B cells (Fig. 3B). Protein kinase B (PKB/AKT) activation is dependent on plasma membrane translocation and phosphorylation on specific amino acid residues, threonine (thr308) and serine (ser473) of AKT. Phosphorylation of the AKT-thr308 site is achieved by PDK-1, while phosphorylation of AKT-ser473 is directly associated with ILK activation $(16,23,24)$. As expected, exposure of Hep3B cells to hypoxia only led to a timedependent increase in phosphoAKT-ser473 and no change in phosphoAKT-thr308 levels as determined by Western blot analyses (Fig. 3B).

To establish the effect that hypoxia has on ILK kinase activity, Hep3B cells were treated for increasing amounts of time in hypoxia $\left(1 \% \mathrm{O}_{2}\right)$ as indicated (Fig. 3C). Following ILK immunoprecipitation of the treated cell lysates, kinase activity was measured with myelin basic protein as the kinase substrate and $\gamma$-ATP as the phosphate donor. The representative autoradiogram demonstrates an increase in ILK kinase activity as a result of hypoxia. The lower panel shown represents the amount of ILK pulled down by the immunoprecipitation as determined from Western blot analysis of the kinase assay gel.

Integrin-linked kinase contributes to anti-apoptotic mechanisms of Hep3B cells in hypoxia. To further characterize the role of 
A

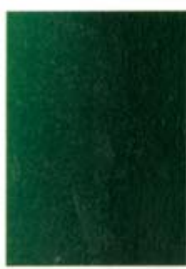

0.03

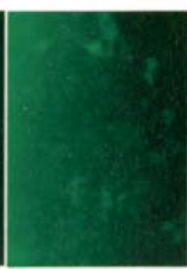

0.3

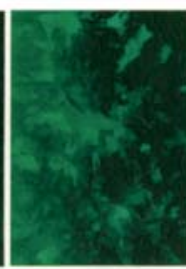

3

MOI of Ad-GFP (p.f.u./cell)

B

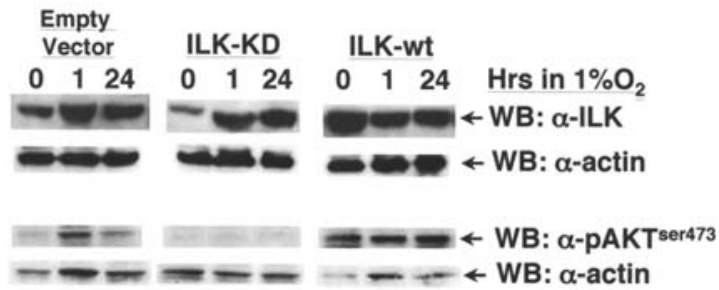

Figure 4. Phosphorylation of AKT serine residue 473 is dampened in Hep3B cells transduced with ILK kinase-dead (E359K) adenovirus vectors compared with cells transduced with wild-type ILK adenovirus vectors or untreated cells. A, micrographs demonstrating transduction efficiency of Hep3B cells treated with increasing adenovirus vectors containing a green fluorescent protein (GFP) reporter gene driven by the herpes cytomegalovirus (CMV) promoter at a multiplicity of infection (MOI) of 0.03-30 plaque forming unit (p.f.u.) per cell as indicated. B, Western blot analysis of cell extracts prepared from transduced Hep3B cells subsequently exposed to $1 \% \mathrm{O}_{2}$ for the times indicated. Approximately thirty micrograms of protein was loaded per well. Arrows alongside the corresponding micrograph indicate the antibodies employed in the Western blot (WB) analysis as described in Fig. 3. Results are representative of at least three experiments performed with independently derived protein extracts.

ILK in hypoxia, Hep3B cells were transduced with adenoviral vectors containing either a kinase-dead dominant-negative mutation of the ilk gene (E359K, Ad-ILK-KD) or wild-type ilk (Ad-ILK-wt) prior to hypoxia treatment and apoptosis analysis. In an attempt to achieve more efficient transgene expression in these cells, adenovirus vectors containing either ILK-wt or ILK-KD genes were developed (AdEasy, Stratagene). For this purpose, the ILK-wt and ILK-KD genes were subcloned into pShuttle-CMV and by double homologous recombination further cloned into an adenovirus (Ad5) backbone (pAdEasy-1). Greater than 90\% transduction was achieved using a multiplicity of infection (MOI) of 30 as determined by fluorescence microscopy of control Ad-GFP Hep3B cell transductions carried out in the same manner as the test Ad-ILK-KD or Ad-ILK-wt transductions (Fig. 4A). Endogenous ILK protein levels were only slightly affected following transduction with Ad-ILK-KD or Ad-ILK-wt when compared to untreated controls as measured by Western blot analysis (Fig. 4B). However, AKT phosphorylation was predictably affected, that is, no change was observed in vector control samples. ILK-KD transduced samples had barely detectable levels and ILK-wt transduced samples demonstrated increased levels of phospho-AKT.

To implicate ILK in Hep3B anti-apoptotic mechanisms, apoptosis in the adenovirus vector-transduced Hep3B cells
A

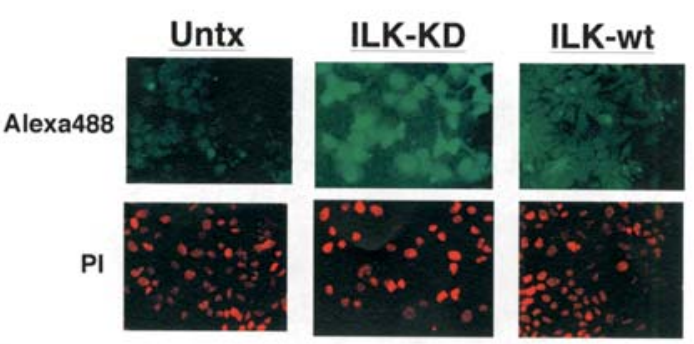

B

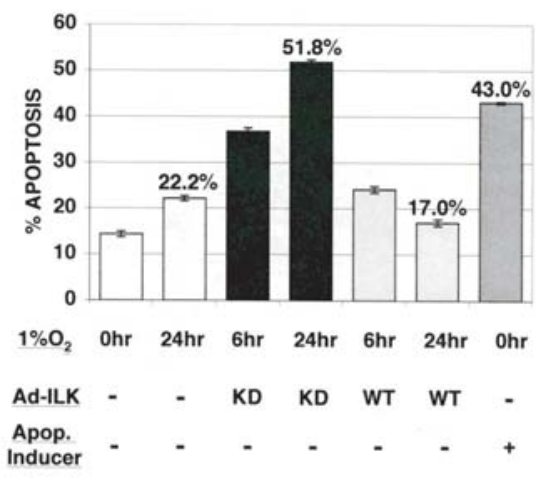

C

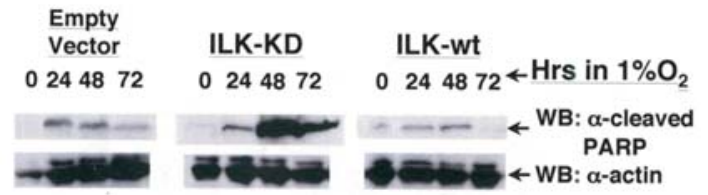

Figure 5. Inhibition of ILK leads to increased apoptosis in hypoxia-treated cells. A, micrographs of TUNEL assay results. Hep3B cells grown on chamber slides were transduced with the adenovirus vectors indicated at an MOI of 30 and exposed to $1 \% \mathrm{O}_{2}$ for $48 \mathrm{~h}$. In this assay, apoptotic nuclei appear green following incubation with fluorescent Alexa Fluor 488-tagged anti-BrDU (Alexa 488) and visualization with UV microscopy. All cell nuclei were stained with propidium iodide (PI) and appear red. Note the smaller amount of red nuclei remaining and dramatic increase in green nuclei following Ad-ILK-KD transduction and hypoxia treatment when compared with other treatments. B, Annexin V flow cytometric analysis of cells treated with adenovirus vectors and exposed to $1 \% \mathrm{O}_{2}$ as indicated. A known apoptotic inducer (30 $\mu \mathrm{M}$ LY294002, $24 \mathrm{~h}$, LC Laboratories, MA) was used as a positive control for this assay and consistently led to $45-50 \%$ apoptosis of treated cells. Apoptosis increased with time in hypoxia for Ad-ILK-KD treated cells as opposed to decreased apoptosis in the Ad-ILK-wt treated cells. Note in particular the dramatic effects of the treatments at $24 \mathrm{~h}$ highlighted by the percentage $(\%)$ of apoptosis numerically represented above the bar graphs. C, Western blot analysis of cleaved poly(ADP)-ribose polymerase (PARP) used as a marker of caspase protease activation. Hep3B cells transduced with the adenovirus vectors indicated above micrographs were exposed to $1 \% \mathrm{O}_{2}$ for the indicated times prior to whole cell lysis and Western blot (WB:) analysis with an antibody specific for the cleaved PARP $(1: 1,000$, Cell Signaling Technology, MA) or B-actin as a loading control. The greatest amount of accumulated cleaved PARP is found in the Ad-ILK-KD samples treated for $48 \mathrm{~h}$ in $1 \% \mathrm{O}_{2}$ as compared to Ad-ILK-wt or untreated control. Results are representative of at least three experiments performed at the least in duplicate.

was measured following hypoxia exposure by several assays including TUNEL assay (Fig. 5A, Molecular Probes, OR), Annexin V-binding (Fig. 5B, ApoAlert assay, BD, Clontech) and detection of immunoreactive cleaved PARP bands (Fig. 5C, Cell Signalling, MA). Similar results were observed for all assays; ILK inhibition achieved by ILK-KD expression led to increased apoptosis following hypoxia exposure, whereas 
A

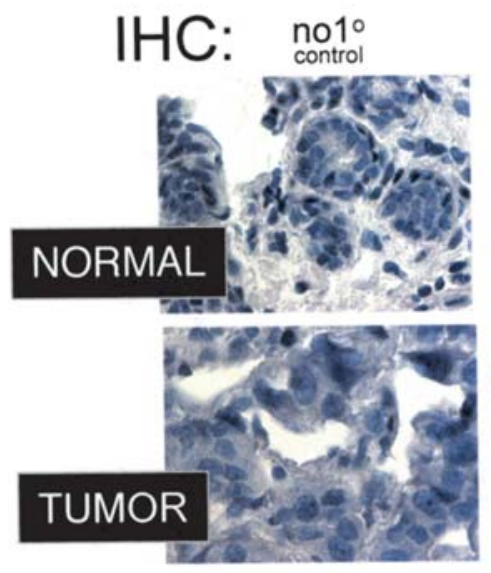

B

\section{Breast}

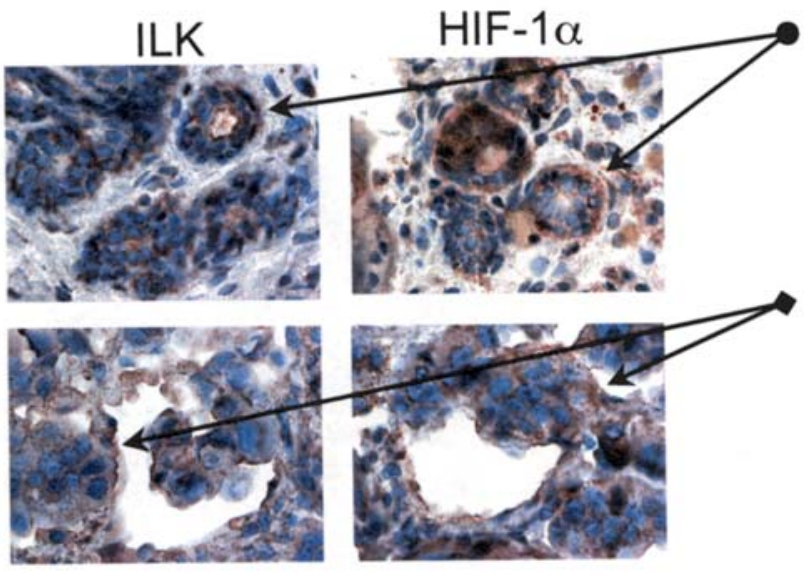

C Kidney

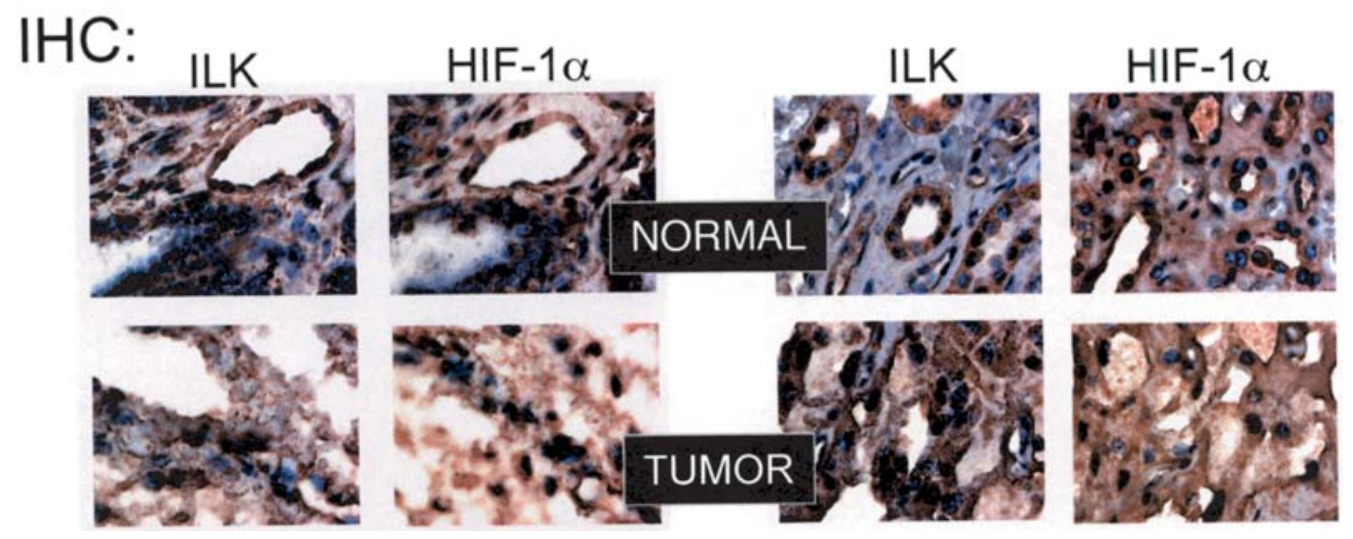

Figure 6. ILK and HIF are co-expressed in many human cancers. Formalin-fixed paraffin-embedded human tissue arrays available for IHC analysis were obtained (Biochain Institute Hayward) and probed with the ILK polyclonal antibody (1:50, cat\#06-592, Upstate Biotechnologies) and HIF-1 $\alpha$ polyclonal antibody (1:200, cat\#sc-12542, Santa Cruz Biotechnologies) for determination of the expression of each of these proteins in a variety of human normal (top panels) and cancerous (bottom panels) tissues, including: A, breast; B, ovary; and C, kidney. IHC staining on corresponding serial sections was achieved with the horseradish peroxidase enhancement method of the LSAB 2 kit (DakoCytomation Corp.). Representative cancer tissues are included for those tissues with the most dramatic results. As a control, the primary antibody was not added to one of the tissue array slides to determine secondary antibody background effects $\left(\right.$ no ${ }^{\circ}$ control). Brown pigmentation reflects antigen expression in the micrographs (x640). Arrows indicate common expression sites for both ILK and HIF$1 \alpha$. It is noteworthy that there is a common expression pattern for these two proteins centered on ductal epithelial cells in both normal and cancerous tissues. By comparison, very little staining is observed in interstitial cells or spaces. In most cases there was a focused expression of both these antigens in normal tissues whereas there is a greater and more diffuse expression for both these antigens in the tumor tissues when compared with the normal.

ILK overexpression achieved by ILK-wt transduction led to increased survival in hypoxia. Note that different exposure times in hypoxia were used to accommodate optimal assay detection times unique to each assay of apoptosis. Shorter time points $(0,6$ and $24 \mathrm{~h})$ were used in ApoAlert assays since this assay measures an early apoptotic event, longer time-points were used for TUNEL and PARP Western blot assays since these tests measure late apoptotic events. The results from these assays indicate that following hypoxia exposure, ILK activation by HIF contributes to survival of Hep3B cells in this harsh low-oxygen environment.

Integrin-linked kinase and HIF are co-expressed in many human cancers. Compelling evidence that ILK plays a critical role in hypoxia anti-apoptotic mechanisms of cancers cells was derived from immunohistochemical analyses of several arrayed human cancer tissues. Formalin-fixed paraffin-embedded tumor tissue arrays available for immunohistochemical analysis were obtained (Biochain Institute Hayward) and probed with the ILK polyclonal antibody (Upstate Biotechnologies) and HIF polyclonal antibody (Santa Cruz Biotechnologies) for determination of the expression of each of these proteins in human cancer tissues. Consistent with this study's concept of HIF-mediated upregulation of ILK, HIF and ILK expression were detected in analogous patterns for arrayed human breast (Fig. 6A), ovarian (Fig. 6B), and kidney cancers (Fig. 6C). Expression patterns differed for these molecules in lung tissues. In those tumors, HIF expression was more prevalent 
than the expression observed for ILK. There was no distinct pattern of HIF and ILK expression noted for the remaining arrayed bladder and uterus tissue samples in this experiment. Additionally, expression of HIF and ILK appeared organized and primarily focused on epithelial cells lining the differing tissue lumen in matched normal specimen. By contrast, the expression of each of these markers in the cancerous tissues was elevated and appeared more diffused throughout the tissues. These results further validate the premise of the study and implicate ILK activation in hypoxia anti-apoptotic mechanisms exploited by human cancers.

\section{Discussion}

Based on cDNA microarray results, ILK emerged as an interesting candidate in hypoxia-mediated anti-apoptotic survival mechanisms employed by cancer cells (1). This notion was confirmed in this study based on the following observations: first, it was determined that the 5 ' promoter region of the ilk gene contains several hypoxia responsive elements (HRE) that were able to specifically bind HIF transcription factor complexes and could be demonstrated to specifically drive HRE-regulated luciferase gene expression in reporter assays in a way similar to the known HIF-regulated gene, erythropoietin; second, ILK protein and kinase activity were induced following hypoxia exposure of cancer cells; third, downstream anti-apoptotic targets of ILK signaling such as phosphoser ${ }^{473} \mathrm{AKT} / \mathrm{PKB}$ were specifically induced following hypoxia treatment; fourth, specific inhibition of ILK led to increased apoptosis of hypoxia-treated Hep3B cancer cells, and lastly, the fact that in vivo expression of HIF might affect ILK expression in human cancers is suggested from co-localized expression of both HIF and ILK within specific cancer tissues and particularly breast, ovary and kidney cancers.

Experiments to identify potential ilk HRE to explain the observed ILK induction in hypoxia by traditional EMSA were successful in confirming HIF-HRE complex formation for nuclear extracts derived from hypoxia-exposed cells (Fig. 2A). Additionally, the three HRE-containing oligoprobes (EpoE, ilk HRE1 or 2) demonstrated equivalent kinetics in binding proteins from nuclear extracts derived following treatment with hypoxia $\left(1 \% \mathrm{O}_{2}\right)$ or treatment with other hypoxia mimetic agents, desferrioxamine (DFO, $100 \mu \mathrm{M}$ ) or cobaltous chloride $(\mathrm{CoCl}, 100 \mu \mathrm{M})$. Similar to other HIFregulated genes, HIF-ilk HRE complex formation peaked following an hour of treatment with hypoxia $\left(1 \% \mathrm{O}_{2}\right)$, DFO or $\mathrm{CoCl}$ and slowly dissipated after $48 \mathrm{~h}$ of either of those treatments (data not shown) (19). Finally, the ILK HREprotein complexes were inhibited but not supershifted by treatment of the nuclear extracts with HIF-1 $\alpha$ antibodies. This effect was not observed with AP-2 shifted protein complexes (data not shown). Taken together these results strongly implicate that HIF proteins specifically bind to ilk HRE.

To further demonstrate that ilk is regulated by HIF transcription factors, three copies of both ilk HRE1 and ilk HRE2 were subcloned into a promoter-containing luciferase reporter vector (Fig. 2B and C). Transient transfection of these constructs resulted in specific luciferase expression. Interestingly, ILK-HRE2 driven luciferase vector appears to function as well as the EpoE HRE luciferase reporter vector (Fig. 2C). However, the effect mediated by the ILK-HRE1 construct was not as robust as the others in hypoxia-induced transactivation of the luciferase gene. The observation that the magnitude of transactivation is not as large for ILK-HRE1, as it is for EpoE HRE or ILK-HRE2 could be explained from the fact that the EpoE HRE reporter vector has five HRE as compared to three in the ILK-HRE vectors. Alternatively, it may be that ILK-HRE1 and -HRE2 are adjacent to the natural ilk gene promoter region to provide synergistic transactivation by the HIF complex or to confer optimal topological conformation for transactivation by HIF. These possibilities were not tested here. The full-length promoter construct for ILK (pILK-Pr) and 3-phosphoinositide-dependent kinase-1 (PDK, pPDK-Pr) containing two and no HRE, respectively, as well as a peroxisome proliferators-activated receptor response element (PPRE) were also tested by this technique (Fig. 2C). Transfection of the Hep3B cells with pILK-Pr consistently resulted in modest hypoxia-, CoClor DFO-mediated induction of the promoter region when compared with empty vector controls or the related PDK1 promoter containing luciferase vectors (16). It is noteworthy that the ilk promoter is likely regulated by multiple factors since this promoter region is specifically regulated by PPAR- $\beta$ and other reports have identified ternary complex factor-1 (TCF-1) sites in this promoter region $(16,25)$. How these components (e.g.- HIF and PPAR- $\beta$ ) might interact and whether these interactions would enhance hypoxia-mediated induction of $i l k$ remains to be determined. Regardless, the data presented here are consistent with the increase in ilk RNA observed by RT-PCR assays of samples obtained following hypoxia exposure of Hep3B cells that we reported previously (1). The sum of these findings strongly supports the concept that HIF specifically mediates ilk HRE transactivation during low oxygen or hypoxia exposure of cells.

Next, the net effect of hypoxia on integrin-linked kinase protein expression in cancer cells was tested by Western blot analysis (Fig. 3A). For this purpose, different human cancer-derived cell lines with functional HIF complexes (Hep3B, RCC-wt) or not (RCC 786-O) were employed. As expected ILK expression following exposure to hypoxia closely correlated with the particular cancer cell's HIF status. ILK protein was induced when the HIF complex was stable and present. We have previously published that ILK protein levels were diminished following hypoxia treatment of Hep3B cells (1). This discrepancy might be explained by the fact that different cell lysis protocols were employed. More recently, commercially available lysis buffers (M-PER, Pierce Biotechnology, IL) used to achieve more reliable results demonstrated by Western blot analysis a consistent increase in ILK immunoreactive bands following hypoxia exposure of the cells. Note that a polyclonal anti-ILK antibody (Upstate Biotechnology) was employed in these studies. This antibody yielded an estimated 59-kDa immunoreactive prominent band following Western blot analysis, however a $50-\mathrm{kDa}$ band has been also reported for ILK in other systems and with different antibody preparations $(26,27)$.

Interestingly, a mutant of ILK (E359K) developed originally as a kinase-dead mutant failed to bind partner focal adhesion-associated proteins (paxillin and actopaxin) and 
thus could not properly localize to focal adhesions to exert its target function. In that study it was reported that expression of this mutant retained in vitro kinase activity (27). In the present study, cell lysates from Hep3B cells transduced with adenoviral vectors developed to contain either the mutant ILK $(\mathrm{E} 359 \mathrm{~K})$ or the wild-type ILK also failed to affect in vitro ILK kinase activity (data not shown). However, we did observe a consistent increase in ILK kinase activity from non-transduced samples obtained following hypoxia exposure (Fig. 3C).

ILK has recently been suggested as an attractive therapeutic cancer target although no definitive study addressing the best cancer types or stages has been reported (14). The results of this study suggest that the most significant impact in an anti-ILK therapy will be achieved in cancers where hypoxia or low-oxygen survival by the tumor has been established. In particular, this study suggests that cancers where HIF stabilization and expression has occurred would be the most vulnerable. In particular, consistent with this study's concept of HIF-mediated upregulation of ILK, HIF and ILK expression were detected in analogous patterns for arrayed human breast (Fig. 6A), ovarian (Fig. 6B), and kidney cancers (Fig. 6C).

Interestingly, Tan et al reported that ILK stimulates the expression of HIF- $1 \alpha$ and subsequently VEGF, one of its downstream target genes important in establishing tumor angiogenesis and blood vessel formation (28). Clearly, a study to establish the critical order of events in the regulation of HIF and ILK, important for tumor establishment and progression, is warranted since HIF biology in cancers appears more complex than initially appreciated as was identified by Raval and colleagues (29). In that study, HIF-1 $\alpha$-mediated gene regulation was compared with HIF- $2 \alpha$-mediated gene regulation in renal carcinoma cells (RCC). Surprisingly, HIF-1 $\alpha$ had contrasting properties to HIF- $2 \alpha$ in the RCC context: HIF- $1 \alpha$ had suppressive tumor interactions whereas HIF- $2 \alpha$ had an enhancing role in tumor xenograft models. Additionally, since Hep3B cells demonstrate greater expression of HIF-2 $\alpha$ than HIF- $1 \alpha$ it is likely that the reported hypoxia-induced anti-apoptotic effects of ILK were driven by HIF- $2 \alpha$. However complex the interaction of ILK and HIF might be, in this study we have identified a mechanism by which a tumor might survive and thrive in an established hypoxia microenvironment. In this scenario, established hypoxia would stabilize HIF transcription factors that would then serve to transactivate ILK expression via its promoter HRE that then activates AKTser473 (and further drives HIF and VEGF expression) that leads to established cell survival. An unexplored and potentially dangerous consequence of ILK kinase activation in hypoxia might be activation of its other downstream transcription factor targets such as NF-kB, AP-1 and B-catenin, all important mediators of cell-cell interactions, migration and invasion. Experiments to confirm these possibilities are underway.

Because of its pinnacle cell signaling role, ILK activation following hypoxia might prove to be an Achilles' heel of tumor survival since specific inhibition of ILK resulted in significant apoptosis of treated cells as determined by three different apoptosis indicators. Inhibition of ILK was reported to lead to increased apoptosis in human breast cancer as well as PTEN-null prostate cancers. A critical role for ILK has also been suggested in colon, gastric, malignant melanomas and Ewing's sarcoma (14). In agreement with these reports, our evidence of co-localization of HIF and ILK in breast and ovary cancers further supports a critical role for ILK in tumorigenesis and potentially adds kidney cancer to this growing list of vulnerable cancers in an anti-ILK therapy. Given its identification as a hypoxia-regulated anti-apoptotic factor, ILK now stands out as a unique target in the successful therapy of many human cancers.

\section{Acknowledgements}

This study was supported by NIH COBRE 1P20RR20152-01, NIH 1 R21 AI056229-01 and a research grant from Cancer Association of Greater New Orleans to A.B.S. There is no conflict of interest with any of the authors. The authors wish to thank Dr Beatrice Desvergne (Universite de Lausanne, Switzerland), Dr James Gnarra (Louisiana State University, LA), Dr Shoukat Dedhar (University of British Columbia, Vancouver, Canada) and their laboratories for their generous contribution of essential reagents in this study. The laboratories of Cindy B. Morris and Deborah E. Sullivan were also invaluable resources in the optimization of some of the assays presented here. We would also like to thank Joanna De Salvo for her assistance in the preparation of figures and Elizabeth Norton for insightful discussions of our work.

\section{References}

1. Scandurro AB, Weldon CW, Figueroa YG, Alam J and Beckman BS: Gene microarray analysis reveals a novel hypoxia signal transduction pathway in human hepatocellular carcinoma cells. Int J Oncol 19: 129-135, 2001.

2. Dedhar S: Cell-substrate interactions and signaling through ILK. Curr Opin Cell Biol 12: 250-256, 2000.

3. Novak A, Hsu SC, Leung-Hagesteijn C, et al: Cell adhesion and the integrin-linked kinase regulate the LEF-1 and beta-catenin signaling pathways. Proc Natl Acad Sci USA 95: 4374-4379, 1998.

4. Troussard AA, Tan C, Yoganathan TN and Dedhar S: Cellextracellular matrix interactions stimulate the AP-1 transcription factor in an integrin-linked kinase- and glycogen synthase kinase 3- dependent manner. Mol Cell Biol 19: 7420-7427, 1999.

5. Lynch DK, Ellis CA, Edwards PA and Hiles ID: Integrin-linked kinase regulates phosphorylation of serine 473 of protein kinase $B$ by an indirect mechanism. Oncogene 18: 8024-8032, 1999.

6. Wu C and Dedhar S: Integrin-linked kinase (ILK) and its interactors: a new paradigm for the coupling of extracellular matrix to actin cytoskeleton and signaling complexes. J Cell Biol 155: 505-510, 2001.

7. Zervas CG and Brown $\mathrm{NH}$ : Integrin adhesion: when is a kinase a kinase? Curr Biol 12: R350-R351, 2002.

8. Wu C, Keightley SY, Leung-Hagesteijn C, et al: Integrin-linked protein kinase regulates fibronectin matrix assembly, E-cadherin expression, and tumorigenicity. J Biol Chem 273: 528-536, 1998.

9. Graff JR, Deddens JA, Konicek BW, et al: Integrin-linked kinase expression increases with prostate tumor grade. Clin Cancer Res 7: 1987-1991, 2001.

10. Chung DH, Lee JI, Kook MC, et al: ILK (beta1-integrin-linked protein kinase): a novel immunohistochemical marker for Ewing's sarcoma and primitive neuroectodermal tumour. Virchows Arch 433: 113-117, 1998.

11. Janji B, Melchior C, Vallar L and Kieffer N: Cloning of an isoform of integrin-linked kinase (ILK) that is upregulated in HT-144 melanoma cells following TGF-beta1 stimulation. Oncogene 19: 3069-3077, 2000.

12. Zhong H, Chiles K, Feldser D, et al: Modulation of hypoxiainducible factor 1alpha expression by the epidermal growth factor/phosphatidylinositol 3-kinase/PTEN/AKT/FRAP pathway in human prostate cancer cells: implications for tumor angiogenesis and therapeutics. Cancer Res 60: 1541-1545, 2000. 
13. Wang $X Q$, Sun $P$ and Paller AS: Inhibition of integrin-linked kinase/protein kinase B/Akt signaling: mechanism for gangliosideinduced apoptosis. J Biol Chem 276: 44504-44511, 2001.

14. Hannigan G, Troussard AA and Dedhar S: Integrin-linked kinase: a cancer therapeutic target unique among its ILK. Nat Rev Cancer 5: 51-63, 2005.

15. Persad S, Attwell S, Gray V, et al: Inhibition of integrin-linked kinase (ILK) suppresses activation of protein kinase B/Akt and induces cell cycle arrest and apoptosis of PTEN-mutant prostate cancer cells. Proc Natl Acad Sci USA 97: 3207-3212, 2000.

16. Di-Poi N, Tan NS, Michalik L, Wahli W and Desvergne B: Antiapoptotic role of PPARbeta in keratinocytes via transcriptional control of the Akt1 signaling pathway. Mol Cell 10: 721-733, 2002.

17. Towbin HST and Gordon J: Electrophoretic transfer of proteins from polyacrylamide gels to nitrocellulose sheets: procedure and some applications. Proc Natl Acad Sci USA 76: 4350-4354, 1979.

18. Hannigan GE, Leung-Hagesteijn C, Fitz-Gibbon L, et al: Regulation of cell adhesion and anchorage-dependent growth by a new beta 1-integrin-linked protein kinase. Nature 379: 91-96, 1996.

19. Wang GL and Semenza GL: Purification and characterization of hypoxia-inducible factor 1. J Biol Chem 270: 1230-1237, 1995.

20. Iliopoulos O, Kibel A, Gray S and Kaelin WG Jr: Tumour suppression by the human von Hippel-Lindau gene product. Nat Med 1: 822-826, 1995

21. Kibel A, Iliopoulos O, De Caprio JA and Kaelin WG Jr: Binding of the von Hippel-Lindau tumor suppressor protein to Elongin B and C. Science 269: 1444-1446, 1995.
22. Lieubeau-Teillet B, Rak J, Jothy S, Iliopoulos O, Kaelin W and Kerbel RS: von Hippel-Lindau gene-mediated growth suppression and induction of differentiation in renal cell carcinoma cells grown as multicellular tumor spheroids. Cancer Res 58: 4957-4962, 1998.

23. Troussard AA, Mawji NM, Ong C, Mui A, St-Arnaud R and Dedhar S: Conditional knock-out of integrin-linked kinase demonstrates an essential role in protein kinase B/Akt activation. J Biol Chem 278: 22374-22378, 2003.

24. Kaneko Y, Kitazato K and Basaki Y: Integrin-linked kinase regulates vascular morphogenesis induced by vascular endothelial growth factor. J Cell Sci 117: 407-415, 2004

25. Melchior C, Kreis S, Janji B and Kieffer N: Promoter characterization and genomic organization of the gene encoding integrinlinked kinase 1. Biochim Biophys Acta 1575: 117-122, 2002.

26. Nikolopoulos SN and Turner CE: Integrin-linked kinase (ILK) binding to paxillin LD1 motif regulates ILK localization to focal adhesions. J Biol Chem 276: 23499-23505, 2001.

27. Nikolopoulos SN and Turner CE: Molecular dissection of actopaxin-integrin-linked kinase-Paxillin interactions and their role in subcellular localization. J Biol Chem 277: 1568-1575, 2002.

28. Tan C, Cruet-Hennequart S, Troussard A, et al: Regulation of tumor angiogenesis by integrin-linked kinase (ILK). Cancer Cell 5: 79-90, 2004.

29. Raval RR, Lau KW, Tran MG, et al: Contrasting properties of hypoxia-inducible factor 1 (HIF-1) and HIF-2 in von HippelLindau-associated renal cell carcinoma. Mol Cell Biol 25: 5675-5686, 2005. 